\title{
ON THE DISTRIBUTION OF THE LIMIT PROPORTION FOR A TWO-COLOR, RANDOMLY REINFORCED URN WITH EQUAL REINFORCEMENT DISTRIBUTIONS
}

\author{
GIACOMO ALETTI* AND \\ CATERINA MAY, ${ }^{*}$ Università degli Studi di Milano \\ PIERCESARE SECCHI, ${ }^{* *}$ Politecnico di Milano
}

\begin{abstract}
We consider a two-color, randomly reinforced urn with equal reinforcement distributions and we characterize the distribution of the urn's limit proportion as the unique continuous solution of a functional equation involving unknown probability distributions on $[0,1]$.

Keywords: Generalized Polya's urn sequence; functional equation in unknown distribution functions; reinforced urn process; randomized response adaptive design of experiments
\end{abstract}

2000 Mathematics Subject Classification: Primary 60B10

Secondary 39B52

\section{Introduction}

An urn initially contains $b>0$ black balls and $w>0$ white balls. The urn is sequentially sampled. At time $n=1,2, \ldots$ a ball is drawn from the urn and its color is observed: if the sampled ball is black it is replaced in the urn together with a random number, $M_{n}$, of balls of color black, if the sampled ball is white it is replaced in the urn together with a random number, $N_{n}$, of balls of color white. The processes $\left\{M_{n}\right\}$ and $\left\{N_{n}\right\}$ are two independent sequences of independent, identically distributed (i.i.d.), nonnegative, and bounded random variables with distributions $\mu$ and $\nu$, respectively. This urn scheme is called a two-color, randomly reinforced urn.

A randomly reinforced urn generates the following interesting stochastic processes: the sequences $\left\{B_{n}\right\}$ and $\left\{W_{n}\right\}$ respectively denote the number of black balls and white balls present in the urn at time $n=1,2, \ldots$; the sequence $\left\{Z_{n}\right\}$ denotes the proportions of black balls in the urn, i.e. for $n=1,2, \ldots$,

$$
Z_{n}=\frac{B_{n}}{B_{n}+W_{n}}
$$

and finally, the sequence $\left\{X_{n}\right\}$ denotes the colors generated by the urn, where $X_{n}$ is 1 or 0 according to whether a black ball or a white ball is drawn from the urn at time $n=1,2, \ldots$ In this paper we mostly focus on the process of urn proportions, $\left\{Z_{n}\right\}$. In [4] it was proved that the sequence $\left\{Z_{n}\right\}$ is eventually a bounded supermartingale or a bounded submartingale and it thus

Received 10 April 2006; revision received 9 January 2007.

* Postal address: Dipartimento di Matematica 'F. Enriques', Università degli Studi di Milano, Via C. Saldini, 50, I-20133 Milano, Italy.

** Postal address: MOX - Dipartimento di Matematica, Politecnico di Milano, Piazza Leonardo da Vinci, 32, I-20133 Milano, Italy. Email address: piercesare.secchi@polimi.it 
converges almost surely to a random limit $Z_{\infty} \in[0,1]$. When the first moment of $\mu$ is strictly greater than the first moment of $v$, the random variable $Z_{\infty}$ is equal to 1 with probability 1 . In the other extreme, [3] showed that if the moments of $\mu$ and $v$ are all equal, i.e. $\mu$ and $v$ coincide, then $Z_{\infty}$ has no point masses. However, except in a few particular cases, the exact distribution of $Z_{\infty}$ is unknown; the only nontrivial case being Polya's urn, where $\mu$ and $v$ are point masses at a nonnegative real number $m$ and $Z_{\infty}$ has distribution $\operatorname{Beta}(b / m, w / m)$. The main result of this paper states that, when $\mu=v$, the distribution of the limit proportion $Z_{\infty}$, regarded as a function of the initial urn composition $(b, w)$, is the unique continuous solution, satisfying some boundary conditions, of a specific functional equation in which the unknowns are distribution functions on $[0,1]$.

The study of the distribution of $Z_{\infty}$ has its origins in the seminal paper of Athreya [1] and stems from theoretical and applicative motives. In [3] and in [4] it was proved that, conditionally on $Z_{\infty}$, the random variables of the sequence of colors $\left\{X_{n}\right\}$ generated by a randomly reinforced urn are asymptotically i.i.d. Bernoulli $\left(Z_{\infty}\right)$; hence the distribution of $Z_{\infty}$ may represent the prior distribution for a Bayesian adopting the randomly reinforced urn scheme as a metaphor for the construction of the statistical model. Moreover, in [4] and in [5] it was stressed that a two-color, randomly reinforced urn implements a sequential, randomized, response-driven design for clinical trials where the experimenter is willing to bias, along the experiment, the allocation probability toward the better treatment; for a well-informed review on urns and response-adaptive, randomized designs see [7]. To be specific, suppose that $\mu$ and $v$ represent the distributions of responses after treatment, say $A$ and $B$, respectively. If the mean response after $A$ is greater than the mean response after $B$, allocating the $n$th patient in the clinical trial to $A$ or $B$ according to the color of the $n$th ball drawn from a two-color, randomly reinforced urn with reinforcements equal to responses after treatments guarantees that patients will be assigned to treatment $A$ with higher and higher probability along the experiment, since $Z_{\infty}=1$ almost surely. Hence, for testing hypotheses about treatment effects, it is important to know the distribution of $Z_{\infty}$ when $\mu=v$, i.e. there is no difference between treatment effects. Indeed, a randomly reinforced urn, where both reinforcement distributions are equal to $\mu$, is the scheme considered in this paper.

After setting the notation and specifying the probabilistic model for a randomly reinforced urn with equal reinforcement distributions in the next section, in Section 3 we introduce the function $\mathcal{F}$ that, given a probability distribution $\mu$, maps any admissible couple, $(b, w)$, in the distribution of the limit proportion of a two-color, randomly reinforced urn with reinforcement distributions equal to $\mu$ and initial composition $(b, w)$. After discussing some interesting properties regarding the class of distributions in the range of $\mathcal{F}$, we close the section with the introduction of a functional equation solved by $\mathcal{F}$ and with the statement of the main result of the paper, namely that $\mathcal{F}$ is the unique continuous solution, satisfying some boundary conditions, of this functional equation. In Section 4 we prove that $\mathcal{F}$ is indeed continuous, while in Section 5 we conclude the proof of the main result by showing that a suitable transformation of $\mathcal{F}$ is the unique fixed point of a certain operator. We conclude the paper with an example in Section 6.

\section{Model specification}

On a rich enough probability space, define two independent sequences $\left\{M_{n}\right\}$ and $\left\{U_{n}\right\}$ of real valued random variables. The variables of the sequence $\left\{M_{n}\right\}$ are i.i.d. with probability distribution $\mu$; the support of $\mu$ is contained in $[0, \beta]$, with $\beta>0$. Moreover, it is assumed that $\mu(\{0\})<1$ in order to avoid the trivial case. The random variables of the sequence $\left\{U_{n}\right\}$ are 
i.i.d. with uniform distribution on $[0,1]$. Finally, let $b$ and $w$ be two nonnegative real numbers such that $b+w>0$.

Set $B_{0}=b, W_{0}=w$, and, for $n \geq 0$, let

$$
\begin{aligned}
B_{n+1} & =B_{n}+M_{n+1} X_{n+1}, \\
W_{n+1} & =W_{n}+M_{n+1}\left(1-X_{n+1}\right),
\end{aligned}
$$

where, for $n=1,2, \ldots$, the variable $X_{n}$ is the indicator of the event

$$
\left\{U_{n} \leq B_{n-1}\left(B_{n-1}+W_{n-1}\right)^{-1}\right\} .
$$

Then the law of the sequence $\left\{\left(B_{n}, W_{n}\right)\right\}$ is that of the stochastic process counting, along time, the number of black and white balls present in a randomly reinforced urn with initial composition $(b, w)$ and reinforcement distributions both equal to $\mu$, whereas the law of the sequence $\left\{X_{n}\right\}$ is that of the process of colors generated by the same urn.

For $n=0,1,2, \ldots$, let

$$
Z_{n}=\frac{B_{n}}{B_{n}+W_{n}} ;
$$

$Z_{n}$ indicates the proportions of black balls in the urn before the $(n+1)$ th ball is sampled. The process $\left\{Z_{n}\right\}$ is a bounded martingale with respect to the filtration $\left\{\sigma\left(X_{1}, M_{1}, \ldots, X_{n-1}, M_{n-1}\right)\right\}$, and it converges almost surely to a random variable $Z_{\infty} \in[0,1]$ (see [3]). Moreover, the distribution of $Z_{\infty}$ has no atoms and is completely determined once the parameters $(b, w, \mu)$ are specified, even though its analytical expression is unknown. We will say that the distribution of $Z_{\infty}$ is that of the limit proportion of a randomly reinforced urn with initial composition $(b, w)$ and reinforcement distributions equal to $\mu$; this paper is focused on this distribution and its properties. Our approach will be to fix the reinforcement distribution $\mu$ and to explore how the distribution of $Z_{\infty}$ varies according to changes in the urn's initial composition $(b, w)$. When the argument requires it, we will draw the reader's attention to the dependency of the law of the stochastic elements generated by the urn on its initial composition $(b, w)$ using the obvious notation $B_{n}(b, w), W_{n}(b, w), X_{n}(b, w), Z_{n}(b, w)$, and $Z_{\infty}(b, w)$.

Pemantle [6] introduced the following time-dependent version of Polya's urn. Let $F:\{1,2, \ldots\} \rightarrow[0, \infty)$ be any function and consider an urn initially containing $b \geq 0$ black balls and $w \geq 0$ white balls, with $b+w>0$. The urn is sequentially sampled; at time $n=1,2, \ldots$ a ball is drawn from the urn and reintroduced into the urn together with $F(n)$ balls of the same color. Pemantle studied the behavior of the sequence of the successive proportions, $V_{n}$, of black balls in the urn. The link between Pemantle's urn and our two-color, randomly reinforced urn with equal reinforcement distributions is evident. In fact, let $\mathcal{M}$ be the sigma-field generated by the random variables $M_{1}, M_{2}, \ldots$ Given $\mathcal{M}$, the conditional law of the process $\left\{\left(B_{n}, W_{n}\right)\right\}$ (and therefore also the conditional laws of the processes $\left\{X_{n}\right\}$ and $\left\{Z_{n}\right\}$ ) is the same as that of the process counting the number of black and white balls in Pemantle's urn once we define his reinforcement function $F$ by setting $F(n)=M_{n}$ for $n=1,2, \ldots$ In the next sections we will make use of the following two results proved in [6].

Theorem 2.1. For any function $F$, the sequence of successive proportions $\left\{V_{n}\right\}$ of black balls in Pemantle's urn directed by $F$ is a martingale converging almost surely to a random variable $V \in[0,1]$. If $F$ is not the null function and it is bounded by some constant $\beta>0$, then $V$ has no atoms in $[0,1]$. 
Theorem 2.2. Consider two Pemantle's urns; both urns have initial composition equal to $(b, w)$. The first urn is directed by the function $F^{(1)}$ and the second urn is directed by the function $F^{(2)}$. Let $\left\{V_{n}^{(1)}\right\}$ and $\left\{V_{n}^{(2)}\right\}$ represent the sequences of successive proportions of black balls in the two urns. If, for all $n=1,2, \ldots$,

$$
\frac{F^{(1)}(n)}{b+w+\sum_{i=1}^{n-1} F^{(1)}(i)} \geq \frac{F^{(2)}(n)}{b+w+\sum_{i=1}^{n-1} F^{(2)}(i)},
$$

then $\mathrm{E}\left[h\left(V_{n+1}^{(1)}\right)\right] \geq \mathrm{E}\left[h\left(V_{n+1}^{(2)}\right)\right]$ for all convex $h:[0,1] \rightarrow[0,2]$.

\section{The function $\mathcal{F}$ and the characteristic equation}

Fix a probability distribution $\mu$ on the interval $[0, \beta]$, with $\beta>0$. Let $S=[0, \infty) \times[0, \infty) \backslash$ $\{(0,0)\}$ and indicate with $\mathcal{P}([0,1])$ the space of distribution functions with support in $[0,1]$; define

$$
\mathcal{F}: S \rightarrow \mathcal{P}([0,1])
$$

to be the function that maps any couple $(b, w)$ of nonnegative real numbers with positive sum in the distribution $\mathcal{F}(b, w)$ of the limit proportion $Z_{\infty}(b, w)$ of a randomly reinforced urn with initial composition $(b, w)$ and reinforcement distributions equal to $\mu$.

For $x \in \mathbb{R}$, let $\delta_{x}$ be the distribution of the point mass at $x$.

Theorem 3.1. (i) $\mathcal{F}(b, 0)=\delta_{1}$ for all $b>0$.

(ii) $\mathcal{F}(0, w)=\delta_{0}$ for all $w>0$.

(iii) For all $(b, w) \in S$,

$$
\mathcal{F}(b, w)(x)+\mathcal{F}(w, b)(1-x)=1 \text { for } x \in \mathbb{R} .
$$

(iv) For all $c \geq 0$ and all $(b, w) \in S$ such that $b /(b+w)=c$,

$$
\int x \mathcal{F}(b, w)(\mathrm{d} x)=c,
$$

i.e. the distribution $\mathcal{F}(b, w)$ has constant mean equal to $c$ along the line

$$
\frac{b}{b+w}=c \text {. }
$$

Proof. Properties (i) and (ii) are trivial to prove. To prove (iii) note that the distribution of the limit proportion of a randomly reinforced urn with reinforcement distributions equal to $\mu$ and initial composition equal to $(b, w)$ must be the same as the distribution of one minus the limit proportion of the same urn when the initial composition is $(w, b)$, i.e. the distribution of $Z_{\infty}(b, w)$ is the same as the distribution of $1-Z_{\infty}(w, b)$; now (iii) follows since the distribution of $Z_{\infty}$ has no point masses (see [3]). Finally, property (iv) is true because, for all $(b, w)$, the sequence $\left\{Z_{n}(b, w)\right\}$ is a bounded martingale converging to $Z_{\infty}(b, w)$ (see [3]) and thus

$$
\mathrm{E}\left[Z_{\infty}(b, w)\right]=Z_{0}(b, w)=\frac{b}{b+w} .
$$


Endow the space $S$ with the lower-right-quadrant order relationship such that $(b, w) \preceq(\bar{b}, \bar{w})$ if and only if $b \leq \bar{b}$ and $w \geq \bar{w}$; give $\mathcal{P}([0,1])$ the stochastic order such that $G \leq_{s t} H$ if and only if $1-G(x) \leq 1-H(x)$ for all $x \in[0,1]$. The proof of the next result uses, for the first time, a coupling argument that will frequently appear in the rest of the paper.

Theorem 3.2. $\mathcal{F}$ is monotonic.

Proof. We need to prove that if $(b, w)$ and $(\bar{b}, \bar{w})$ are states of $S$ such that $(b, w) \preceq(\bar{b}, \bar{w})$, then

$$
\mathcal{F}(b, w) \leq_{s t} \mathcal{F}(\bar{b}, \bar{w}) .
$$

Consider two different randomly reinforced urns. The first urn has initial composition $(b, w)$ and generates the counting process $\left\{\left(B_{n}, W_{n}\right)\right\}$ according to the dynamics described in (2.1). Analogously, the second urn has initial composition $(\bar{b}, \bar{w})$ and generates the counting process $\left\{\left(\bar{B}_{n}, \bar{W}_{n}\right)\right\}$. The urns are coupled in the sense that the processes $\left\{M_{n}\right\},\left\{U_{n}\right\}$ and $\left\{\bar{M}_{n}\right\},\left\{\bar{U}_{n}\right\}$ appearing in (2.1) for the definition of $\left\{\left(B_{n}, W_{n}\right)\right\}$ and $\left\{\left(\bar{B}_{n}, \bar{W}_{n}\right)\right\}$, respectively, are identical; i.e. we assume that

$$
\mathrm{P}\left[M_{n}=\bar{M}_{n}, U_{n}=\bar{U}_{n} \text { for all } n\right]=1 .
$$

Note that $b /(b+w) \leq \bar{b} /(\bar{b}+\bar{w})$; hence $U_{1} \leq \bar{b} /(\bar{b}+\bar{w})$ if $U_{1} \leq b /(b+w)$ and

$$
\mathrm{P}\left[\left(B_{1}, W_{1}\right) \preceq\left(\bar{B}_{1}, \bar{W}_{1}\right)\right]=1 .
$$

By induction on $n$,

$$
\mathrm{P}\left[\left(B_{n}, W_{n}\right) \preceq\left(\bar{B}_{n}, \bar{W}_{n}\right) \text { for all } n\right]=1
$$

and thus

$$
\mathrm{P}\left[Z_{\infty}(b, w) \leq Z_{\infty}(\bar{b}, \bar{w})\right]=1 .
$$

Since the support of $\mu$ is bounded above by $\beta$, it is natural to conjecture that, when the initial number of balls in the urn is large, the limit proportion $Z_{\infty}$ will be close to its mean value; in fact, we will prove the conjecture by means of Theorem 3.3. For $(b, w) \in S$, let $B(b / \beta, w / \beta)$ indicate a random variable with distribution $\operatorname{Beta}(b / \beta, w / \beta)$ on $[0,1]$.

Lemma 3.1. For every $j \geq 1$ and $(b, w) \in S$,

$$
\mathrm{E}\left[Z_{\infty}^{j}(b, w)\right] \leq \mathrm{E}\left[B^{j}\left(\frac{b}{\beta}, \frac{w}{\beta}\right)\right] .
$$

Proof. Let $j \geq 1$ and $N \geq 1$. Given $M_{1}=m_{1}, M_{2}=m_{2}, \ldots$, and $M_{N}=m_{n}, \ldots$ note that

$$
\mathrm{E}\left[Z_{N}^{j}(b, w) \mid M_{1}=m_{1}, M_{2}=m_{2}, \ldots\right]=\mathrm{E}\left[V_{N}^{j}\right],
$$

where the sequence $V_{1}, V_{2}, \ldots$ denotes the proportion of black balls in Pemantle's urn with initial composition $(b, w)$ and reinforcement directed by $F$, with $F(n)=m_{n}$ for $n \leq N$ and $F(n)=0$ for $n>N$. We now use a trick learned in [6]. If it is not the case that $F(1) \geq F(2) \geq F(3) \geq \ldots$, let $F^{(1)}(n)=F(n)$ for every $n$ except for two indices $k \geq 1$ and $k+1$, where $F^{(1)}(k)=F(k+1)>F(k)=F^{(1)}(k+1)$, and consider Pemantle's urn with initial composition $(b, w)$ and reinforcements directed by $F^{(1)}$. Then Proposition 2 of [6] proves that

$$
\mathrm{E}\left[h\left(V_{n}\right)\right] \leq \mathrm{E}\left[h\left(V_{n}^{(1)}\right)\right]
$$


for all $n \geq 1$ and for all convex $h:[0,1] \rightarrow[0,2]$, where $V_{n}^{(1)}$ indicates the successive proportions of black balls in the urn directed by $F^{(1)}$; that is, $V_{n}$ and $V_{n}^{(1)}$ are convex ordered. By repeatedly applying this result and by setting $h(x)=x^{j}$ for $x \in[0,1]$, we obtain

$$
\mathrm{E}\left[V_{N}^{j}\right] \leq \mathrm{E}\left[\left(V_{N}^{(2)}\right)^{j}\right]
$$

where, for $n=1,2, \ldots, V_{n}^{(2)}$ are the successive proportions of black balls in Pemantle's urn with reinforcements directed by a function $F^{(2)}$ with the same values as $F$ but rearranged in descending order. Finally, let the sequence $\bar{V}_{1}, \bar{V}_{2}, \ldots$ denote the proportions of black balls in Polya's urn with initial composition $(b, w)$ and constant reinforcement equal to $\beta$; Theorem 2.2 implies that

$$
\mathrm{E}\left[\left(V_{N}^{(2)}\right)^{j}\right] \leq \mathrm{E}\left[\bar{V}_{N}^{j}\right]
$$

Let $\mathcal{M}$ be the sigma-field generated by $M_{1}, M_{2}, \ldots$ Then (3.2), (3.3), and (3.4) prove that, for $N \geq 1$ and $(b, w) \in S$,

$$
\mathrm{E}\left[Z_{N}^{j}(b, w) \mid \mathcal{M}\right] \leq \mathrm{E}\left[\bar{V}_{N}^{j}\right]
$$

Equation (3.1) now follows by computing the expected values on both sides of the above inequality and applying the dominated convergence theorem.

Theorem 3.3. For every $\eta>0$ and $\varepsilon>0$, there exists $K=K(\eta, \varepsilon)$ such that

$$
\mathrm{P}\left[\left|Z_{\infty}(b, w)-\frac{b}{b+w}\right|>\eta\right]<\varepsilon
$$

if $b+w>K$.

Proof. Let $(b, w) \in S$ and $\eta>0$. Then

$$
\begin{aligned}
\mathrm{P}\left[\left|Z_{\infty}(b, w)-\frac{b}{b+w}\right|>\eta\right] & \leq \frac{1}{\eta^{2}} \operatorname{var}\left(Z_{\infty}(b, w)\right) \\
& \leq \frac{1}{\eta^{2}} \frac{b w}{(b+w)^{2}} \frac{\beta}{b+w+\beta} \\
& \leq \frac{1}{\eta^{2}} \frac{1}{4} \frac{\beta}{b+w} ;
\end{aligned}
$$

the first inequality is Chebichev's while the second one follows from (3.1) with $j=2$ and the fact that the expected value of $Z_{\infty}(b, w)$ is $b /(b+w)$. The theorem is thus proved by setting $K=\beta\left(4 \eta^{2} \varepsilon\right)^{-1}$.

Theorem 3.3 has interesting consequences in applications, for instance, when we want to approximate the distribution of $Z_{\infty}$ by means of a Monte Carlo simulation. In fact, given $\varepsilon, \eta>0$, let

$$
\tau(\varepsilon, \eta)=\inf \left\{n \geq 0: B_{n}+W_{n}>K(\eta, \varepsilon)\right\} .
$$

Since $\mu$ is not concentrated on $0, \tau(\varepsilon, \eta)$ is finite almost surely; the next corollary states that little is lost by approximating the distribution of $Z_{\infty}$ with that of $Z_{\tau(\varepsilon, \eta)}$. Its proof follows from Theorem 3.3 and the observation that the process $\left\{\left(B_{n}, W_{n}\right)\right\}$ is Markov. Hence, the strong Markov property holds and implies that, given the sigma-field generated by the stopping time $\tau(\varepsilon, \eta)$, the conditional law of the process

$$
\left(\left(B_{\tau(\varepsilon, \eta)}, W_{\tau(\varepsilon, \eta)}\right),\left(B_{\tau(\varepsilon, \eta)+1}, W_{\tau(\varepsilon, \eta)+1}\right), \ldots\right)
$$




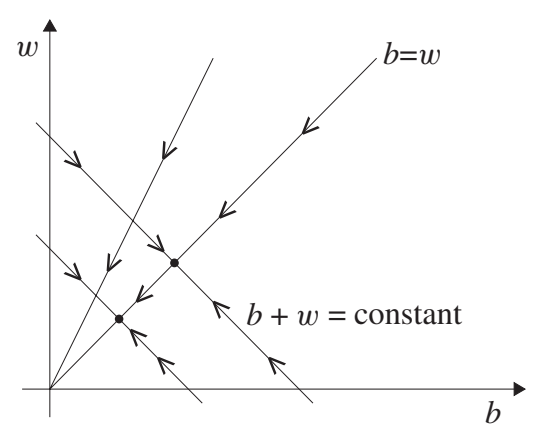

FIGURE 1: The variance of $Z_{\infty}(b, w)$.

is that of the process counting the successive number of balls of color black and white, respectively, in a randomly reinforced urn with reinforcement distributions equal to $\mu$ and initial composition equal to $\left(B_{\tau(\varepsilon, \eta)}, W_{\tau(\varepsilon, \eta)}\right)$.

Corollary 3.1. For all $(b, w) \in S$ and $\varepsilon, \eta>0$,

$$
\mathrm{P}\left[\left|Z_{\infty}(b, w)-Z_{\tau(\varepsilon, \eta)}(b, w)\right|>\eta\right]<\varepsilon .
$$

Remark 3.1. If $\mu$ has support contained in $[\alpha, \beta]$ with $\alpha>0$, then

$$
\mathrm{P}\left[\tau(\varepsilon, \eta) \leq \frac{\beta}{4 \alpha \eta^{2} \varepsilon}+1\right]=1 .
$$

Finally, we consider the variance of $Z_{\infty}(b, w)$. The next result states that for $b+w=$ constant, the variance of $Z_{\infty}(b, w)$ reaches its maximum value when $b=w$, and that for $b=($ constant $)(w)$, i.e. along the lines from the origin, the variance of $Z_{\infty}(b, w)$ decreases when $w$ increases; see Figure 1.

Theorem 3.4. (i) For every $(b, w) \in S$,

$$
\operatorname{var}\left(Z_{\infty}(b, w)\right) \leq \operatorname{var}\left(Z_{\infty}\left(\frac{b+w}{2}, \frac{b+w}{2}\right)\right) .
$$

(ii) For every $(b, w) \in S$ and $t>1$,

$$
\operatorname{var}\left(Z_{\infty}(b, w)\right) \geq \operatorname{var}\left(Z_{\infty}(t b, t w)\right) .
$$

Proof. To prove (i), set $\Delta^{2} Z_{n+1}=Z_{n+1}^{2}-Z_{n}^{2}$ for $n=0,1, \ldots$; we claim that, for $(b, w) \in S$,

$$
\mathrm{E}\left[\Delta^{2} Z_{n+1}(b, w)\right] \leq \mathrm{E}\left[\Delta^{2} Z_{n+1}\left(\frac{b+w}{2}, \frac{b+w}{2}\right)\right] .
$$

Since $\operatorname{var}\left(Z_{n+1}\right)=\mathrm{E}\left[\sum_{i=0}^{n} \Delta^{2} Z_{i+1}\right]$, from (3.5) it follows that

$$
\operatorname{var}\left(Z_{n+1}(b, w)\right) \leq \operatorname{var}\left(Z_{n+1}\left(\frac{b+w}{2}, \frac{b+w}{2}\right)\right)
$$

for all $n$; letting $n \rightarrow \infty$ we obtain (i) from the dominated convergence theorem. 
In order to prove (3.5), let $\mathcal{M}$ be the sigma-field generated by $M_{1}, M_{2}, \ldots$, and compute

$$
\begin{aligned}
\mathrm{E}\left[Z_{n+1}^{2} \mid \mathcal{M}\right] & =\mathrm{E}\left[\mathrm{E}\left[Z_{n+1}^{2} \mid Z_{n}, \mathcal{M}\right] \mid \mathcal{M}\right] \\
& =\mathrm{E}\left[Z_{n}^{2}+H_{n+1}^{2} Z_{n}-H_{n+1}^{2} Z_{n}^{2} \mid \mathcal{M}\right],
\end{aligned}
$$

where

for $n=1,2, \ldots$ Hence,

$$
H_{n}=H_{n}(b, w)=\frac{M_{n}}{b+w+\sum_{i=1}^{n} M_{i}}
$$

$$
\mathrm{E}\left[Z_{n+1}^{2}-Z_{n}^{2} \mid \mathcal{M}\right]=H_{n+1}^{2} \mathrm{E}\left[Z_{n}-Z_{n}^{2} \mid \mathcal{M}\right] .
$$

For $n=0,1, \ldots$, set $W_{n}=\mathrm{E}\left[Z_{n}-Z_{n}^{2} \mid \mathcal{M}\right]$. Theorem 2.1 states that the proportion of black balls in Pemantle's time-dependent version of Polya's urn is a martingale, thus, $\mathrm{E}\left[Z_{n+1} \mid \mathcal{M}\right]=$ $\mathrm{E}\left[Z_{n} \mid \mathcal{M}\right]$ for $n=0,1,2, \ldots$ Hence, (3.6) implies that, for all $n$,

$$
W_{n+1}=W_{n}\left(1-H_{n+1}^{2}\right)
$$

and then

$$
W_{n}=W_{0} \prod_{i=1}^{n}\left(1-H_{i}^{2}\right) .
$$

Hence, (3.7) can be rewritten as

$$
\mathrm{E}\left[Z_{n+1}^{2}-Z_{n}^{2} \mid \mathcal{M}\right]=H_{n+1}^{2} W_{0} \prod_{i=1}^{n}\left(1-H_{i}^{2}\right) .
$$

Taking expectations, we find that

$$
\mathrm{E}\left[\Delta^{2} Z_{n+1}\right]=W_{0} \mathrm{E}\left[H_{n+1}^{2} \prod_{i=1}^{n}\left(1-H_{i}^{2}\right)\right] .
$$

Finally, to obtain (3.5) from (3.8), it is sufficient to note that

$$
H_{n}(b, w)=H_{n}\left(\frac{b+w}{2}, \frac{b+w}{2}\right)
$$

and that

$$
W_{0}(b, w)=\frac{b}{b+w}\left(1-\frac{b}{b+w}\right)
$$

reaches its maximum value when $b=w$.

To prove (ii), consider two urns in which the first has initial composition $(b, w) \in S$ and the second has initial composition $(t b, t w)$, with $t>1$. The urns are coupled in the sense that, as in the proof of Theorem 3.2, the same sequences $\left\{M_{n}\right\}$ and $\left\{U_{n}\right\}$ generate the urns successive compositions by means of (2.1). Note that, for $n=1,2, \ldots$,

$$
\frac{M_{n}}{b+w+\sum_{i=1}^{n-1} M_{i}} \geq \frac{M_{n}}{t(b+w)+\sum_{i=1}^{n-1} M_{i}} .
$$

By conditioning on $\mathcal{M}$ and setting $h(x)=(x-b /(b+w))^{2}$ for $x \in[0,1]$, it follows, from Theorem 2.2, that

$$
\mathrm{E}\left[h\left(Z_{n}(b, w)\right) \mid \mathcal{M}\right] \geq \mathrm{E}\left[h\left(Z_{n}(t b, t w)\right) \mid \mathcal{M}\right] .
$$

Now (ii) follows by taking expectations and limits. 
By conditioning on $X_{1}$ and $M_{1}$ and computing the expected values, we see that $\mathcal{F}$ must satisfy the following condition: for all $(b, w) \in S$,

$$
\mathcal{F}(b, w)=\frac{b}{b+w} \int_{[0, \beta]} \mathcal{F}(b+k, w) \mu(\mathrm{d} k)+\frac{w}{b+w} \int_{[0, \beta]} \mathcal{F}(b, w+k) \mu(\mathrm{d} k) .
$$

We call (3.9) the characteristic equation of a randomly reinforced urn with reinforcement distributions equal to $\mu$. The following question arises naturally: does (3.9) characterize the class of distributions for limit proportions of randomly reinforced urns with reinforcement distributions equal to $\mu$ and initial compositions varying in $S$ ? In other words, is the function $\mathcal{F}$ introduced in this section the unique map from $S$ to $\mathcal{P}([0,1])$ that solves the functional equation (3.9)? Without further conditions, the answer to this question is no; for instance, any constant function mapping $S$ into a fixed element of $\mathcal{P}([0,1])$ is a solution of (3.9), although, what we already know about $\mathcal{F}$ implies that $\mathcal{F}$ is not a constant function. The main result of this paper states that $\mathcal{F}$ is the unique continuous solution of (3.9) satisfying some boundary conditions. In order to have a precise statement of the theorem, consider $S$ to be a subset of the space $\mathbb{R}^{2}$ with the Euclidean metric and endow $\mathcal{P}([0,1])$ with the Wasserstein metric defined, for all $F, G \in \mathcal{P}([0,1])$, as

$$
d_{W}(F, G)=\int_{0}^{1}|F(x)-G(x)| \mathrm{d} x .
$$

Theorem 3.5. The function $\mathcal{F}$ is the unique solution of (3.9) among the continuous functions $g: S \rightarrow \mathcal{P}[0,1]$ satisfying the following three conditions:

(a) $\mathcal{g}(0, w)=\delta_{0}$ for $w>0$;

(b) $g(b, 0)=\delta_{1}$ for $b>0$;

(c) for every $\varepsilon>0$, there exists a $K=K(\varepsilon)$ such that

$$
d_{W}\left(g(b, w), \delta_{b /(b+w)}\right)<\varepsilon
$$

$$
\text { if } b+w>K \text {. }
$$

We already know that $\mathcal{F}$ satisfies conditions (a) and (b) of the theorem: in the next section we will prove that $\mathcal{F}$ is indeed continuous on $S$ and satisfies condition (c). Then, in Section 5, through a suitable transformation of the space $S$ and the function $\mathcal{F}$, we will prove that $\mathcal{F}$ is the unique continuous solution of (3.9) satisfying (a)-(c).

Remark 3.2. When modeling our two-color, randomly reinforced urn with reinforcement distributions equal to $\mu$, we assumed that $\mu(\{0\})<1$. For all Borel subsets $B$ of $\mathbb{R}$, define

$$
\nu(B)=\frac{\mu(B \cap(0, \beta])}{1-\mu(\{0\})} ;
$$

hence, $v$ is the conditional distribution of $M_{1}$, given that $M_{1}>0$. Note that, $\mathcal{F}: S \rightarrow \mathcal{P}([0,1])$ solves (3.9) if and only if

$[1-\mu(\{0\})] \mathcal{F}(b, w)=\frac{b}{b+w} \int_{(0, \beta]} \mathcal{F}(b+k, w) \mu(\mathrm{d} k)+\frac{w}{b+w} \int_{(0, \beta]} \mathcal{F}(b, w+k) \mu(\mathrm{d} k)$, 
i.e. if and only if

$$
\mathcal{F}(b, w)=\frac{b}{b+w} \int_{(0, \beta]} \mathcal{F}(b+k, w) v(\mathrm{~d} k)+\frac{w}{b+w} \int_{(0, \beta]} \mathcal{F}(b, w+k) v(\mathrm{~d} k)
$$

for all $(b, w) \in S$. In light of Theorem 3.5, we can thus say that $\mathcal{F}$ is also the unique solution of (3.11) among the continuous functions $g: S \rightarrow \mathscr{P}([0,1])$ satisfying conditions (a)-(c) of the theorem.

\section{4. $\mathcal{F}$ is continuous}

Before proceeding to prove that $\mathcal{F}$ is continuous, we recall that the distance $d_{W}$, introduced in (3.10), metrizes the weak convergence in $\mathcal{P}([0,1])$. Moreover, by the KantorovichRubinstein theorem,

$$
d_{W}(F, G)=\inf \{\mathrm{E}[|X-Y|]: X \sim F, Y \sim G\}
$$

where the infimum is taken over all joint distributions for $(X, Y)$ with marginals equal to $F$ and $G$, respectively; see [2] for a review on this and other distances for probability distribution functions.

\section{Theorem 4.1. $\mathcal{F}$ is continuous on $S$.}

Proof. (i) To prove that $\mathcal{F}$ is continuous on the axes consider a point $(0, \bar{w})$ with $\bar{w}>0$ and note that $\mathcal{F}(0, \bar{w})=\delta_{0}$ by Theorem 3.1(ii). For $(b, w) \in S$,

$$
d_{W}(\mathcal{F}(b, w), \mathcal{F}(0, \bar{w}))=\int_{0}^{1}(1-\mathcal{F}(b, w)(x)) \mathrm{d} x=\mathrm{E}\left[Z_{\infty}(b, w)\right]=\frac{b}{b+w}
$$

Hence, $\lim _{(b, w) \rightarrow(0, \bar{w})} d_{W}(\mathcal{F}(b, w), \mathcal{F}(0, \bar{w}))=0$. This proves that $\mathcal{F}$ is continuous on the axis $b=0$; by symmetry, $\mathcal{F}$ is also continuous on the axis $w=0$.

(ii) To prove that $\mathcal{F}$ is continuous at the inner points of $S$ consider a point $(\bar{b}, \bar{w}) \in S$ with $\bar{b}>0$ and $\bar{w}>0$. For $(b, w) \in S$, compare two randomly reinforced urns with reinforcement distributions equal to $\mu$ and initial compositions $(\bar{b}, \bar{w})$ and $(b, w)$. As in the proof of Theorem 3.2, the two urns are coupled in the sense that the same processes $\left\{M_{n}\right\}$ and $\left\{U_{n}\right\}$ generate both $\left\{\left(B_{n}(b, w), W_{n}(b, w)\right)\right\}$ and $\left\{\left(B_{n}(\bar{b}, \bar{w}), W_{n}(\bar{b}, \bar{w})\right)\right.$ according to the dynamics described in $(2.1)$; let $Z_{\infty}(b, w)$ and $Z_{\infty}(\bar{b}, \bar{w})$ be the limit proportions for the two urns.

From (4.1) and the triangular inequality, it follows that

$$
\begin{aligned}
d_{W}(\mathcal{F}(b, w), \mathcal{F}(\bar{b}, \bar{w})) \leq & \mathrm{E}\left[\left|Z_{\infty}(b, w)-Z_{\infty}(\bar{b}, \bar{w})\right|\right] \\
\leq & \mathrm{E}\left[\left|Z_{\infty}(b, w)-Z_{N}(b, w)\right|\right]+\mathrm{E}\left[\left|Z_{\infty}(\bar{b}, \bar{w})-Z_{N}(\bar{b}, \bar{w})\right|\right] \\
& +\mathrm{E}\left[\left|Z_{N}(b, w)-Z_{N}(\bar{b}, \bar{w})\right|\right]
\end{aligned}
$$

for all $N \geq 1$. 
Next we prove that the two terms of the second line in (4.2) can be taken to be arbitrarily small for large enough $N$. In fact, for all $(b, w) \in S$ and $m \geq 1$,

$$
\begin{aligned}
\mathrm{E}\left[\mid Z_{\infty}\right. & \left.(b, w)-Z_{m}(b, w) \mid\right] \\
& \leq \mathrm{E}^{1 / 2}\left[\left|Z_{\infty}(b, w)-Z_{m}(b, w)\right|^{2}\right] \\
& =\mathrm{E}^{1 / 2}\left[\mathrm{E}\left(\left|Z_{\infty}(b, w)-Z_{m}(b, w)\right|^{2} \mid\left(B_{1}, W_{1}\right), \ldots,\left(B_{m}, W_{m}\right)\right)\right] \\
& =\mathrm{E}^{1 / 2}\left[\mathrm{E}\left[Z_{\infty}^{2}\left(B_{m}, W_{n}\right) \mid\left(B_{m}, W_{m}\right)\right]-\left(\frac{B_{m}}{B_{m}+W_{m}}\right)^{2}\right] \\
& \leq \mathrm{E}^{1 / 2}\left[\frac{B_{m} W_{m}}{\left(B_{m}+W_{m}\right)^{2}} \frac{\beta}{B_{m}+W_{m}+\beta}\right] ;
\end{aligned}
$$

the last equality is true because the process $\left\{\left(B_{n}, W_{n}\right)\right\}$ is Markov and, moreover,

$$
\mathrm{E}\left[Z_{\infty}(b, w) \mid\left(B_{1}, W_{1}\right), \ldots,\left(B_{m}, W_{m}\right)\right]=B_{m}\left(B_{m}+W_{m}\right)^{-1}
$$

the last inequality follows from Lemma 3.1 as in the proof of Theorem 3.3. Since $\mu(\{0\})<1$,

$$
\mathrm{P}\left[\lim _{n \rightarrow \infty} \sum_{i=1}^{n} M_{i}=\infty\right]=1 .
$$

Therefore, for every $\varepsilon>0$ and $K>0$, there exists an $m=m(K, \varepsilon)$ such that

$$
\mathrm{P}\left[\sum_{i=1}^{m} M_{i}>K\right] \geq 1-\varepsilon^{2}
$$

For $(b, w) \in S$, let $F$ be the event that is true when

$$
B_{m}(b, w)+W_{m}(b, w)+\beta=\beta+b+w+\sum_{i=1}^{m} M_{i}>K
$$

then $\mathrm{P}(F) \geq 1-\varepsilon^{2}$. Set $K=\beta / 4 \varepsilon^{2}$ and $N=m(K, \varepsilon)$. Then, for all $(b, w) \in S$,

$$
\begin{aligned}
\mathrm{E}\left[\left|Z_{\infty}(b, w)-Z_{N}(b, w)\right|\right] \leq & \mathrm{E}^{1 / 2}\left[\frac{B_{N} W_{N}}{\left(B_{N}+W_{N}\right)^{2}} \frac{\beta}{B_{N}+W_{N}+\beta}\right] \\
= & \left(\mathrm{E}\left[\frac{B_{N} W_{N}}{\left(B_{N}+W_{N}\right)^{2}} \frac{\beta}{B_{N}+W_{N}+\beta} ; F\right]\right. \\
& \left.+\mathrm{E}\left[\frac{B_{N} W_{N}}{\left(B_{N}+W_{N}\right)^{2}} \frac{\beta}{B_{N}+W_{N}+\beta} ; F^{c}\right]\right)^{1 / 2} \\
\leq & \left(\frac{1}{4} \mathrm{E}\left[\frac{\beta}{B_{N}+W_{N}+\beta} ; F\right]+\mathrm{P}\left(F^{c}\right)\right)^{1 / 2} \\
\leq & \left(\varepsilon^{2} \mathrm{P}(F)+\mathrm{P}\left(F^{c}\right)\right)^{1 / 2} \\
\leq & \varepsilon \sqrt{2},
\end{aligned}
$$

where the $c$ superscript denotes the complimentary of an event. 
Now consider the term $\mathrm{E}\left[\left|Z_{N}(b, w)-Z_{N}(\bar{b}, \bar{w})\right|\right]$ in $(4.2)$. For $i=1,2, \ldots, N$, let

$$
A_{i}=\left\{X_{i}(b, w) \neq X_{i}(\bar{b}, \bar{w})\right\}
$$

and set $A=\bigcup_{i=1}^{N} A_{i}$. Then

$$
\mathrm{P}\left(A_{1}\right)=\left|\frac{b}{b+w}-\frac{\bar{b}}{\bar{b}+\bar{w}}\right| .
$$

However, for all $\left(b_{0}, w_{0}\right)$ and $\left(b_{1}, w_{1}\right)$ in $S$, note that

$$
\begin{aligned}
\left|\frac{b_{0}}{b_{0}+w_{0}}-\frac{b_{1}}{b_{1}+w_{1}}\right| & \left|\frac{b_{0}}{b_{0}+w_{0}}-\frac{b_{0}}{b_{0}+\max \left(w_{0}, w_{1}\right)}\right| \\
& +\left|\frac{b_{0}}{b_{0}+\max \left(w_{0}, w_{1}\right)}-\frac{\max \left(b_{0}, b_{1}\right)}{\max \left(b_{0}, b_{1}\right)+\max \left(w_{0}, w_{1}\right)}\right| \\
& +\left|\frac{\max \left(b_{0}, b_{1}\right)}{\max \left(b_{0}, b_{1}\right)+\max \left(w_{0}, w_{1}\right)}-\frac{b_{1}}{b_{1}+\max \left(w_{0}, w_{1}\right)}\right| \\
& +\left|\frac{b_{1}}{b_{1}+\max \left(w_{0}, w_{1}\right)}-\frac{b_{1}}{b_{1}+w_{1}}\right| \\
\leq & \frac{\left|w_{0}-\max \left(w_{0}, w_{1}\right)\right|}{b_{0}+w_{0}}+\frac{\left|b_{0}-\max \left(b_{0}, b_{1}\right)\right|}{b_{0}+\max \left(w_{0}, w_{1}\right)} \\
& +\frac{\left|b_{1}-\max \left(b_{0}, b_{1}\right)\right|}{b_{1}+\max \left(w_{0}, w_{1}\right)}+\frac{\left|w_{1}-\max \left(w_{0}, w_{1}\right)\right|}{b_{1}+w_{1}} \\
\leq & \frac{\left|b_{0}-b_{1}\right|+\left|w_{0}-w_{1}\right|}{\min \left(b_{0}+w_{0}, b_{1}+w_{1}\right)} .
\end{aligned}
$$

Hence,

$$
\mathrm{P}\left(A_{1}\right) \leq \frac{|b-\bar{b}|+|w-\bar{w}|}{\min (b+w, \bar{b}+\bar{w})} .
$$

Analogously, for $i=1, \ldots, N-1$,

$$
\begin{aligned}
& \mathrm{P}\left(A_{i+1} \mid\left(\bigcup_{j=1}^{i} A_{j}\right)^{c}\right) \\
& \leq \mathrm{E}\left[\left|Z_{i}(b, w)-Z_{i}(\bar{b}, \bar{w})\right| \mid\left(\bigcup_{j=1}^{i} A_{j}\right)^{c}\right] \\
& \leq \mathrm{E}\left[\frac{\left|B_{i}(b, w)-B_{i}(\bar{b}, \bar{w})\right|+\left|W_{i}(b, w)-W_{i}(\bar{b}, \bar{w})\right|}{\min \left(B_{i}(b, w)+W_{i}(b, w), B_{i}(\bar{b}, \bar{w})+W_{i}(\bar{b}, \bar{w})\right)} \mid\left(\bigcup_{j=1}^{i} A_{j}\right)^{c}\right] \\
& \leq \frac{|b-\bar{b}|+|w-\bar{w}|}{\min (b+w, \bar{b}+\bar{w})}
\end{aligned}
$$

where the second inequality follows from (4.4) and the third inequality holds because, when $\left(\bigcup_{j=1}^{i} A_{j}\right)^{c}$ is true, $\left|B_{i}(b, w)-B_{i}(\bar{b}, \bar{w})\right|=|b-\bar{b}|$ and $\left|W_{i}(b, w)-W_{i}(\bar{b}, \bar{w})\right|=|w-\bar{w}|$, while 
it is always true that $\min \left(B_{i}(b, w)+W_{i}(b, w), B_{i}(\bar{b}, \bar{w})+W_{i}(\bar{b}, \bar{w})\right) \geq \min (b+w, \bar{b}+\bar{w})$. Thus,

$$
\begin{aligned}
\mathrm{P}(A) & =\mathrm{P}\left(A_{1} \cup A_{2} \cup \cdots \cup A_{N}\right) \\
& =\mathrm{P}\left(A_{1} \cup\left(A_{2} \cap A_{1}{ }^{c}\right) \cup \cdots \cup\left(A_{N} \cap\left(A_{1} \cup \cdots \cup A_{N-1}\right)^{c}\right)\right) \\
& \leq \mathrm{P}\left(A_{1}\right)+\mathrm{P}\left(A_{2} \mid A_{1}{ }^{c}\right)+\cdots+\mathrm{P}\left(A_{N} \mid\left(A_{1} \cup \cdots \cup A_{N-1}\right)^{c}\right) \\
& \leq N \frac{|b-\bar{b}|+|w-\bar{w}|}{\min (b+w, \bar{b}+\bar{w})} .
\end{aligned}
$$

Moreover, when $A^{c}$ is true it follows, from a final application of (4.4), that

$$
\begin{aligned}
\left|Z_{N}(b, w)-Z_{N}(\bar{b}, \bar{w})\right| & \leq \frac{\left|B_{N}(b, w)-B_{N}(\bar{b}, \bar{w})\right|+\left|W_{N}(b, w)-W_{N}(\bar{b}, \bar{w})\right|}{\min \left(B_{N}(b, w)+W_{N}(b, w), B_{N}(\bar{b}, \bar{w})+W_{N}(\bar{b}, \bar{w})\right)} \\
& \leq \frac{|b-\bar{b}|+|w-\bar{w}|}{\min (b+w, \bar{b}+\bar{w})} .
\end{aligned}
$$

Therefore,

$$
\begin{aligned}
& \mathrm{E}\left[\left|Z_{N}(b, w)-Z_{N}(\bar{b}, \bar{w})\right|\right] \\
& \quad=\mathrm{E}\left[\left|Z_{N}(b, w)-Z_{N}(\bar{b}, \bar{w})\right| ; A^{c}\right]+\mathrm{E}\left[\left|Z_{N}(b, w)-Z_{N}(\bar{b}, \bar{w})\right| ; A\right] \\
& \quad \leq(1+N) \frac{|b-\bar{b}|+|w-\bar{w}|}{\min (b+w, \bar{b}+\bar{w})} .
\end{aligned}
$$

To complete the proof, let $\varepsilon>0$. From (4.2), (4.3), and (4.5) it follows that there exists an $N=N(\varepsilon)$ such that

$$
d_{W}(\mathcal{F}(b, w), \mathcal{F}(\bar{b}, \bar{w})) \leq \varepsilon 2 \sqrt{2}+(1+N) \frac{|b-\bar{b}|+|w-\bar{w}|}{\min (b+w, \bar{b}+\bar{w})}
$$

Hence,

$$
\lim _{(b, w) \rightarrow(\bar{b}, \bar{w})} d_{W}(\mathcal{F}(b, w), \mathcal{F}(\bar{b}, \bar{w})) \leq \varepsilon 2 \sqrt{2}
$$

Since $\varepsilon$ is arbitrary, this proves that

$$
\lim _{(b, w) \rightarrow(\bar{b}, \bar{w})} d_{W}(\mathcal{F}(b, w), \mathcal{F}(\bar{b}, \bar{w}))=0 .
$$

An immediate corollary of Theorem 3.3 is that $\mathcal{F}$ is continuous at the points of the projective line corresponding to the directions $b(b+w)^{-1}$, if we set $\mathcal{F}$ equal to $\delta_{b /(b+w)}$ in these points. Figure 2 illustrates the next result.

Corollary 4.1. For every $\varepsilon>0$ there exists a $K=K(\varepsilon)$ such that if $b+w>K$, then

$$
d_{W}\left(\mathcal{F}(b, w), \delta_{b /(b+w)}\right)<\varepsilon .
$$

We conclude the section by describing the behavior of $\mathscr{F}$ near the origin of $\mathbb{R}^{2}$. Note that $\mathcal{F}$ cannot be extended with continuity in $(0,0)$. In fact $\mathcal{F}(b, w)$ has constant mean, $c$, along the points $(b, w) \in S$ such that $b(b+w)^{-1}=c$; hence, the weak limit of $\mathcal{F}(b, w)$ for $(b, w) \rightarrow(0,0)$ does not exist. 


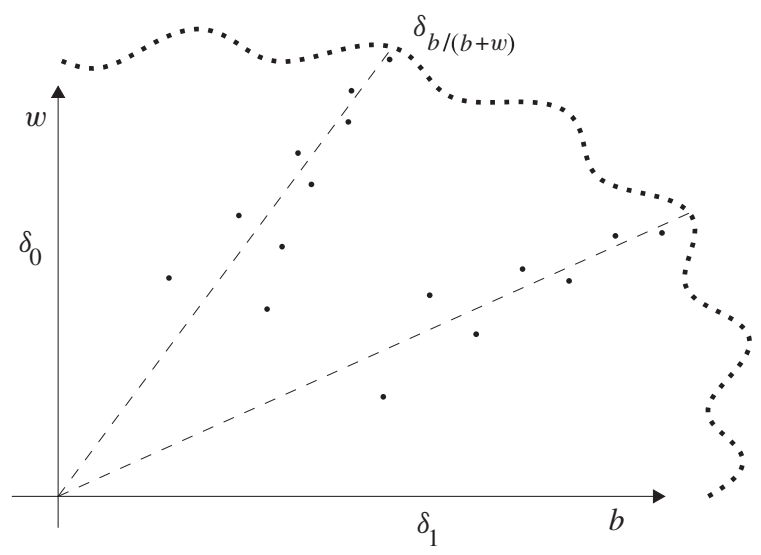

FIGURE 2: Continuity of $\mathcal{F}$ on the points of the projective line.

Theorem 4.2. For every $\varepsilon>0$ there exists a neighborhood $U_{\varepsilon}$ of $(0,0)$ such that

$$
d_{W}\left(\mathscr{F}(b, w), \frac{b}{b+w} \delta_{1}+\frac{w}{b+w} \delta_{0}\right)<\varepsilon
$$

for $(b, w) \in U_{\varepsilon} \cap S$.

Proof. As stated in Remark 3.2, $\mathcal{F}$ solves (3.9) if and only if, for all $(b, w) \in S$,

$$
\mathcal{F}(b, w)=\frac{b}{b+w} \int_{(0, \beta]} \mathcal{F}(b+k, w) v(\mathrm{~d} k)+\frac{w}{b+w} \int_{(0, \beta]} \mathcal{F}(b, w+k) v(\mathrm{~d} k),
$$

where $v$ is the conditional probability distribution of $M_{1}$, given that $M_{1}>0$. Since the support of $v$ is contained in $(0, \beta]$, for every $\varepsilon>0$ there exists an $\alpha>0$ such that $v((0, \alpha])<\varepsilon / 2$. Let $b<\alpha \varepsilon / 2$ and $w<\alpha \varepsilon / 2$ and set $y=b(b+w)^{-1}$. Then

$$
\begin{aligned}
d_{W}(\mathcal{F} & \left.(b, w), y \delta_{1}+(1-y) \delta_{0}\right) \\
& =\int_{0}^{1}|\mathcal{F}(b, w)(x)-(1-y)| \mathrm{d} x \\
& =\int_{0}^{1}\left|y \int \mathcal{F}(b+k, w)(x) v(\mathrm{~d} k)+(1-y) \int \mathcal{F}(b, w+k)(x) v(\mathrm{~d} k)-(1-y)\right| \mathrm{d} x \\
& \leq \int_{0}^{1} y \int \mathcal{F}(b+k, w)(x) v(\mathrm{~d} k) \mathrm{d} x+\int_{0}^{1}(1-y) \int(1-\mathcal{F}(b, w+k)(x)) v(\mathrm{~d} k) \mathrm{d} x \\
& =y \int \frac{w}{b+w+k} v(\mathrm{~d} k)+(1-y) \int \frac{b}{b+w+k} v(\mathrm{~d} k) \\
& \leq y\left(\frac{\varepsilon}{2}+\frac{w}{b+w+\alpha}\right)+(1-y)\left(\frac{\varepsilon}{2}+\frac{b}{b+w+\alpha}\right) \\
& \leq y\left(\frac{\varepsilon}{2}+\frac{\alpha \varepsilon / 2}{\alpha}\right)+(1-y)\left(\frac{\varepsilon}{2}+\frac{\alpha \varepsilon / 2}{\alpha}\right) \\
& =\varepsilon .
\end{aligned}
$$

Remark 4.1. Theorem 4.2 implies that $\mathcal{F}$ can be extended with continuity in the origin $(0,0)$ along the lines $b(b+w)^{-1}=$ constant. 


\section{5. $\mathcal{F}$ is the unique solution of the characteristic equation}

Given the probability distribution $\mu$, in order to describe the distribution of the next state of a randomly reinforced urn with reinforcement distributions equal to $\mu$ we need to know either the current number of black and white balls contained in the urn or, equivalently, the current total number of balls in the urn and the proportion of black balls. This fact and the behavior of the function $\mathcal{F}$ near the origin and at $\infty$ suggest a transformation of the state space $S$ according to the map

$$
\tau:\left\{\begin{array}{l}
x=\frac{1}{b+w}, \\
y=\frac{b}{b+w} .
\end{array}\right.
$$

Let $S^{*}=[0, \infty) \times[0,1]$. For $(x, y) \in(0, \infty) \times[0,1]$, define

$$
\mathcal{F}^{*}(x, y)=\mathcal{F}\left(\tau^{-1}(x, y)\right)
$$

and set

$$
\mathcal{F}^{*}(0, y)=\delta_{y}
$$

for $y \in[0,1]$.

Theorem 5.1. $\mathcal{F}^{*}$ is continuous on $S^{*}$.

Proof. Because of Theorem 4.1, we need to prove the continuity of $\mathcal{F}^{*}$ only in the states $(0, y) \in S^{*}$, with $y \in[0,1]$. Let $\bar{y} \in[0,1]$ and consider the state $(0, \bar{y})$; the triangular inequality implies that, for every $(x, y) \in S^{*}$,

$$
d_{W}\left(\mathcal{F}^{*}(x, y), \mathcal{F}^{*}(0, \bar{y})\right)=d_{W}\left(\mathcal{F}^{*}(x, y), \delta_{\bar{y}}\right) \leq d_{W}\left(\mathcal{F}^{*}(x, y), \delta_{y}\right)+d_{W}\left(\delta_{\bar{y}}, \delta_{y}\right) .
$$

However,

$$
d_{W}\left(\mathcal{F}^{*}(x, y), \delta_{y}\right)=d_{W}\left(\mathcal{F}\left(\frac{y}{x}, \frac{1-y}{x}\right), \delta_{y}\right),
$$

and Corollary 4.1 implies that this quantity converges to 0 when $x \rightarrow 0$, whereas

$$
d_{W}\left(\delta_{\bar{y}}, \delta_{y}\right)=\int_{0}^{1}\left|\delta_{\bar{y}}(x)-\delta_{y}(x)\right| \mathrm{d} x=|y-\bar{y}|
$$

converges to 0 when $y \rightarrow \bar{y}$.

The function $\mathcal{F}^{*}$ maps any $(x, y) \in(0, \infty) \times[0,1]$ in the distribution $\mathcal{F}\left(y x^{-1},(1-y) x^{-1}\right)$ of the limit proportion $Z_{\infty}$ of a randomly reinforced urn with reinforcement distributions equal to $\mu$ and initial composition equal to $\left(y x^{-1},(1-y) x^{-1}\right)$. The results of Section 3 allow some instructive descriptions of the distribution $\mathcal{F}^{*}(x, y)$. For instance, along the horizontal lines $y=$ constant, the mean of $\mathcal{F}^{*}(x, y)$ is constant while the variance increases with $x$. Along the vertical lines $x=$ constant, the variance of $\mathcal{F}^{*}(x, y)$ increases as $y$ moves from 0 to $\frac{1}{2}$ and decreases as $y$ moves from $\frac{1}{2}$ to 1 , reaching its maximum value at $y=\frac{1}{2}$. Moreover, for $(x, y) \in S^{*}$,

$$
\mathcal{F}^{*}(x, 0)=\delta_{0}, \quad \mathcal{F}^{*}(x, 1)=\delta_{1}, \quad \text { and } \quad \mathcal{F}^{*}(0, y)=\delta_{y} ;
$$

see Figure 3 for an illustration. 


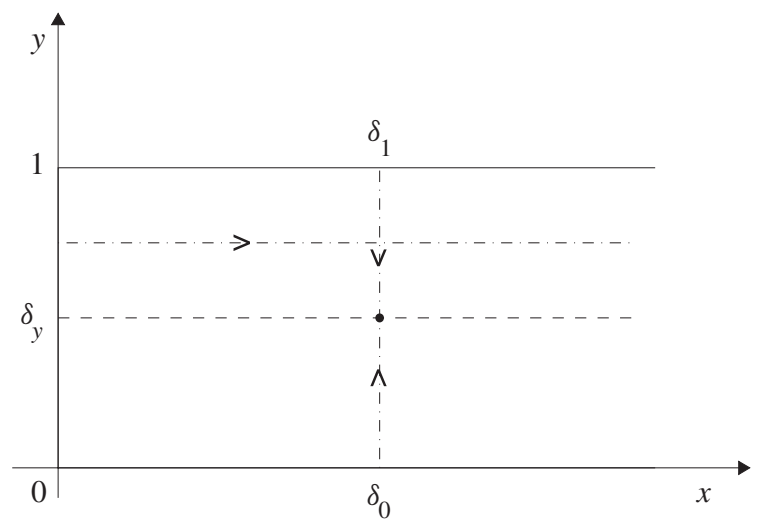

FIGURE 3: Boundary values of $\mathcal{F}^{*}$ and the variance of $\mathcal{F}^{*}(x, y)$.

Finally, note that $\mathcal{F}^{*}$ satisfies the characteristic equation

$$
\begin{aligned}
\mathcal{F}^{*}(x, y)= & y \int \mathcal{F}^{*}\left(\frac{x}{1+k x}, \frac{y+k x}{1+k x}\right) \mu(\mathrm{d} k) \\
& +(1-y) \int \mathcal{F}^{*}\left(\frac{x}{1+k x}, \frac{y}{1+k x}\right) \mu(\mathrm{d} k) .
\end{aligned}
$$

Let $\mathbb{C}\left(S^{*}\right)$ be the space of continuous functions $g: S^{*} \rightarrow \mathcal{P}([0,1])$ such that, for every $(x, y) \in S^{*}$,

$$
g(x, 0)=\delta_{0}, \quad g(x, 1)=\delta_{1}, \quad \text { and } \quad g(0, y)=\delta_{y} .
$$

For $(x, y) \in S^{*}$ and $g \in \mathbb{C}\left(S^{*}\right)$, define

$$
A^{*}(g)(x, y)=y \int g\left(\frac{x}{1+k x}, \frac{y+k x}{1+k x}\right) \mu(\mathrm{d} k)+(1-y) \int g\left(\frac{x}{1+k x}, \frac{y}{1+k x}\right) \mu(\mathrm{d} k) ;
$$

then $A^{*}(g)(x, y) \in \mathcal{P}([0,1])$. Note that, for every $g \in \mathbb{C}\left(S^{*}\right)$, the function $A^{*}(g) \in \mathbb{C}\left(S^{*}\right)$; hence we may regard $A^{*}$ as an operator mapping $\mathbb{C}\left(S^{*}\right)$ into $\mathbb{C}\left(S^{*}\right)$.

Theorem 5.2. $\mathcal{F}^{*}$ is the unique fixed point of $A^{*}$.

Proof. For $c \in(0, \infty)$, let $S_{c}^{*}=[0, c] \times[0,1]$ and consider the space $\mathbb{C}\left(S_{c}^{*}\right)$ of the continuous functions $g: S_{c}^{*} \rightarrow \mathcal{P}([0,1])$ such that, for every $(x, y) \in S^{*}$,

$$
g(x, 0)=\delta_{0}, \quad g(x, 1)=\delta_{1}, \quad \text { and } \quad g(0, y)=\delta_{y} .
$$

Set $A_{c}^{*}$ to be the restriction of $A^{*}$ to $\mathbb{C}\left(S_{c}^{*}\right)$; note that $A_{c}^{*}$ maps $\mathbb{C}\left(S_{c}^{*}\right)$ into $\mathbb{C}\left(S_{c}^{*}\right)$. Let $\mathcal{F}_{c}^{*}$ be the restriction of $\mathcal{F}^{*}$ to $S_{c}^{*}$. Note that, for every $c>0, \mathcal{F}_{c}^{*}$ is a fixed point of $A_{c}^{*}$ since $\mathcal{F}^{*}$ satisfies (5.1). If $\mathcal{F}_{c}^{*}$ is the unique fixed point of $A_{c}^{*}$, then the theorem is proved because $S^{*}=\bigcup_{c>0} S_{c}^{*}$.

By way of contradiction, assume that, for $c>0$, there exists a $g^{*} \in \mathbb{C}\left(S_{c}^{*}\right)$ that is a fixed point of $A_{c}^{*}$ different from $\mathcal{F}_{c}^{*}$. Then we claim there exists an $(\bar{x}, \bar{y}) \in S_{c}^{*}$ such that

$$
d_{W}\left(\mathcal{F}_{c}^{*}(\bar{x}, \bar{y}), \mathcal{G}^{*}(\bar{x}, \bar{y})\right)>d_{W}\left(A_{c}^{*}\left(\mathcal{F}_{c}^{*}\right)(\bar{x}, \bar{y}), A_{c}^{*}\left(\mathcal{G}^{*}\right)(\bar{x}, \bar{y})\right),
$$

which contradicts the assumption that both $\mathcal{F}_{c}^{*}$ and $\mathcal{g}^{*}$ are fixed points of $A_{c}^{*}$. 
To prove (5.2), for $(x, y) \in S_{c}^{*}$, define

$$
\Phi_{\mathcal{F}_{c}^{*}, \mathcal{F}^{*}}(x, y)=d_{W}\left(\mathcal{F}_{c}^{*}(x, y), \mathcal{g}^{*}(x, y)\right) .
$$

Observe that $\Phi_{\mathcal{F}_{c}^{*}, \mathcal{G}^{*}}$ is a continuous function defined on the compact set $S_{c}^{*}$. Moreover, $\Phi_{\mathcal{F}_{c}^{*}, q^{*}} \geq 0$ and, for $(x, y) \in S_{c}^{*}$,

$$
\Phi_{\mathcal{F}_{c}^{*}, \mathcal{G}^{*}}(x, 0)=\Phi_{\mathcal{F}_{c}^{*}, g^{*}}(x, 1)=\Phi_{\mathcal{F}_{c}^{*}, g^{*}}(0, y)=0 .
$$

However, $\Phi_{\mathcal{F}_{c}^{*}, g^{*}} \not \equiv 0$ since, by assumption, $g^{*} \neq \mathcal{F}_{c}^{*}$. Let

$$
M=\max \left\{\Phi_{\mathcal{F}_{c}^{*}}, \mathcal{F}^{*}(x, y):(x, y) \in S_{c}^{*}\right\} .
$$

Then $\Phi_{\mathcal{F}_{c}^{*}, \mathcal{F}^{*}}^{-1}(M)$ is a closed subset of $(0, c] \times(0,1)$. Indicate with $\Pi_{1}: \mathbb{R}^{2} \rightarrow \mathbb{R}$ the projection on the first coordinate defined by setting $\Pi_{1}(z, w)=z$ for $(z, w) \in \mathbb{R}^{2}$. Since $\Pi_{1}$ is an open map, $\Pi_{1}\left(\Phi_{\mathcal{F}_{c}^{*}, q^{*}}^{-1}(M)\right)$ is a closed subset of $(0, c]$. Let

$$
\bar{x}=\min \left\{x: x \in \Pi_{1}\left(\Phi_{\mathcal{F}_{c}^{*}, \mathcal{G}^{*}}^{-1}(M)\right)\right\}
$$

and $\bar{y} \in[0,1]$ such that $\Phi_{\mathcal{F}_{c}^{*}, g^{*}}(\bar{x}, \bar{y})=M$. The point $(\bar{x}, \bar{y})$ makes (5.2) true. In fact, firstly note that, for $(x, y) \in S_{c}^{*}$ with $x<\bar{x}, \Phi_{\mathcal{F}_{c}^{*}, \mathcal{G}^{*}}(x, y)<M$. Then compute

$$
\begin{aligned}
d_{W} & \left(A_{c}^{*}\left(\mathcal{F}_{c}^{*}\right)(\bar{x}, \bar{y}), A_{c}^{*}\left(\mathcal{g}^{*}\right)(\bar{x}, \bar{y})\right) \\
= & \int_{0}^{1}\left|A_{c}^{*}\left(\mathcal{F}_{c}^{*}\right)(\bar{x}, \bar{y})(t)-A_{c}^{*}\left(\mathcal{g}^{*}\right)(\bar{x}, \bar{y})(t)\right| \mathrm{d} t \\
= & \int_{0}^{1} \mid \bar{y} \int \mathcal{F}_{c}^{*}\left(\frac{\bar{x}}{1+k \bar{x}}, \frac{\bar{y}+k \bar{x}}{1+k \bar{x}}\right)(t) \mu(\mathrm{d} k)+(1-\bar{y}) \int \mathcal{F}_{c}^{*}\left(\frac{\bar{x}}{1+k \bar{x}}, \frac{\bar{y}}{1+k \bar{x}}\right)(t) \mu(\mathrm{d} k) \\
& \quad-\bar{y} \int g^{*}\left(\frac{\bar{x}}{1+k \bar{x}}, \frac{\bar{y}+k \bar{x}}{1+k \bar{x}}\right)(t) \mu(\mathrm{d} k) \\
& \quad-(1-\bar{y}) \int \mathcal{g}^{*}\left(\frac{\bar{x}}{1+k \bar{x}}, \frac{\bar{y}}{1+k \bar{x}}\right)(t) \mu(\mathrm{d} k) \mid \mathrm{d} t \\
\leq & \bar{y} \int \Phi_{\mathcal{F}_{c}^{*}, g^{*}}\left(\frac{\bar{x}}{1+k \bar{x}}, \frac{\bar{y}+k \bar{x}}{1+k \bar{x}}\right) \mu(\mathrm{d} k)+(1-\bar{y}) \int \Phi_{\mathcal{F}_{c}^{*}, \mathcal{g}^{*}}\left(\frac{\bar{x}}{1+k \bar{x}}, \frac{\bar{y}}{1+k \bar{x}}\right) \mu(\mathrm{d} k) \\
< & \bar{y} M_{+}(1-\bar{y}) M \\
= & \Phi_{\mathcal{F}_{c}^{*}, g^{*}(\bar{x}, \bar{y})} \\
= & d_{W}\left(\mathcal{F}_{c}^{*}(\bar{x}, \bar{y}), g^{*}(\bar{x}, \bar{y})\right) .
\end{aligned}
$$

This proves (5.2) and shows that $\mathcal{F}_{c}^{*}$ is the unique fixed point of $A_{c}^{*}$.

Theorem 3.5 is now easy to obtain.

Proof of Theorem 3.5. Theorem 4.1 proves that $\mathcal{F}$ is continuous, while $\mathcal{F}$ satisfies conditions (a)-(c) because of Theorem 3.1(i), Theorem 3.1(ii), and Corollary 4.1. Assume that $g: S \rightarrow \mathcal{P}([0,1])$ is another continuous solution of (3.9) for which (a)-(c) are true; then, as in Theorem 5.1, show that $g^{*}: S^{*} \rightarrow \mathcal{P}([0,1])$ defined by setting $\mathcal{G}^{*}(x, y)=g_{(}\left(\tau^{-1}(x, y)\right)$ for $(x, y) \in(0, \infty) \times[0,1]$, and $\mathcal{g}^{*}(0, y)=\delta_{y}$ for $y \in[0,1]$, is continuous on $S^{*}$ and satisfies (a)-(c). Verify, moreover, that $A^{*}\left(g^{*}\right)=\mathcal{G}^{*}$, i.e. that $\mathcal{g}^{*}$ solves (5.1). Hence, Theorem 5.2 proves that $\mathcal{G}^{*}=\mathcal{F}^{*}$ and thus $\mathcal{G}=\mathcal{F}$. 


\section{An example}

When $\mu=\delta_{\beta}$, the point mass at a given $\beta>0$, our two-color, randomly reinforced urn with reinforcement distributions equal to $\mu$ becomes Polya's urn and the unique continuous solution of (3.9) satisfying conditions (a)-(c) of Theorem 3.5 is

$$
\mathcal{F}(b, w)=\operatorname{Beta}\left(\frac{b}{\beta}, \frac{w}{\beta}\right)
$$

for $(b, w) \in S$, where, for $c, d>0, \operatorname{Beta}(c, d)$ is the Beta distribution on [0,1] whereas we define $\operatorname{Beta}(c, 0)=\delta_{1}$ and $\operatorname{Beta}(0, d)=\delta_{0}$.

Now let $\mu=p \delta_{0}+(1-p) \delta_{\beta}$ with $p \in(0,1)$ and $\beta>0$. Then, by Remark 3.2, $\operatorname{Beta}(b / \beta, w / \beta)$ is again the unique continuous solution of (3.9) satisfying conditions (a)-(c) of Theorem 3.5.

The next step is to assume that $\mu=p \delta_{\alpha}+(1-p) \delta_{\beta}$ with $0<\alpha<\beta$ integers and $p \in(0,1)$. Let $M=p \alpha+(1-p) \beta$ be the mean of $\mu$. A result of [1] implies that the solution $\mathcal{F}(b, w)$ of (3.9) determined by Theorem 3.5 cannot be equal to $\operatorname{Beta}(b / M, w / M)$ for $(b, w) \in S$. A tentative guess could be

$$
\mathcal{F}(b, w)=p \operatorname{Beta}\left(\frac{b}{\alpha}, \frac{w}{\alpha}\right)+(1-p) \operatorname{Beta}\left(\frac{b}{\beta}, \frac{w}{\beta}\right)
$$

for $(b, w) \in S$. This $\mathcal{F}$ does not solve (3.9). In fact, for $(b, w) \in S$, the second moment of $\mathcal{F}(b, w)$, defined as in (6.1), coincides with the second moment of the right member of (3.9) if and only if

$$
\frac{b w p(1-p)(\beta-\alpha)^{2}}{(b+w)(b+w+\alpha)(b+w+\beta)(b+w+\alpha+\beta)}=0
$$

for all $(b, w) \in S$; but this cannot happen since $0<\alpha<\beta$ and $p \in(0,1)$. Hence, in this case the analytical expression of $\mathcal{F}(b, w)$ is still unknown, although interesting approximations for it can be found by simulations, as in [3].

\section{References}

[1] Athreya, K. B. (1969). On a characteristic property of Polya's urn. Studia Sci. Math. Hungarica 4, 31-35.

[2] GibBs, A. L. AND Su, F. E. (2002). On choosing and bounding probability metrics. Internat. Statist. Rev. 70, 419-435.

[3] May, C., Paganoni, A. M. and Secchi, P. (2005). On a two color generalized Pólya urn. Metron 63, $115-134$.

[4] Muliere, P., Paganoni, A. M. And Secchi, P. (2006). A two-color, randomly reinforced urn. J. Statist. Planning Infer. 136, 1853-1874.

[5] Paganoni, A. M. And Secchi, P. (2007). A numerical study for comparing two response-adaptive designs for continuous treatment effects. To appear in Statist. Meth. Appl.

[6] Pemantle, R. (1990). A time-dependent version of Pólya's urn. J. Theoret. Prob. 3, 627-637.

[7] Rosenberger, W. F. (2002). Randomized urn models and sequential design. Sequential Anal. 21, 1-28. 